\title{
И.С. ФИШМАН,
}

Самарский филиал Российской академии народного хозяйства и государственной службы при Президенте РФ (Самара, Российская Федерация; e-mail: fishman-is@ranepa.ru)

\section{КОММЕРЦИАЛИЗАЦИЯ ИННОВАЦИЙ В ПРАКТИКЕ ЗАРУБЕЖНЫХ УНИВЕРСИТЕТОВ}

УДК: 33

https://doi.org/10.22394/2410-132X-2020-6-3-159-168

I Аннотация. Показано, что большинство зарубежных исследователей считают университетские структуры

I важным и экономически устойчивым механизмом передачи новых технологий промышленности, эти структуры

I ускоряют экономическое развитие территорий путем создания новых наукоемких рабочих мест, и увеличивают

I налоговые поступления в местные бюджеты. Благодаря государственному финансированию и общественной

I поддержке, университетские исследовательские структуры демонстрируют более высокую выживаемость по

- сравнению с фирмами, аффилированными корпоративным организациям.

I Аффилированные университетам структуры очень активно используют государственные программы помощи,

1 дольше остаются обитателями бизнес - инкубаторов и являются бенефициарами фондов, поддерживающих

I научно-исследовательские и опытно-конструкторские разработки, их охотно финансируют частные венчурные

I фонды. Они являются более инновативными, чем фирмы, связанные с корпоративными организациями,

I имеют большее количество защищенных патентами изобретений, демонстрируют более высокий уровень

I выживаемости и более высокую оценку ликвидности, однако имеют более низкую рентабельность.

I Связанные с университетами инновационные фирмы могут коммерциализировать как конкретные изобретения,

I так и более неявное знание, полученное в результате научных исследований. Отмечено, что самой большой

I сложностью для таких фирм является преобразование технологических активов в рыночные предложения.

I Ключевые слова: аффилированные университетам структуры, трансфер технологий/инноваций,

I коммерциализация инноваций университетами, центры по передаче технологий, университетские инкубаторы,

I совместные исследовательские центры, внутренний фонд посевного финансирования

Для цитирования: Фишман И.С. Коммерциализация инноваций в практике зарубежных университетов. Экономика I науки. 2020; 6(3):159-168. https://doi.org/10.22394/2410-132X-2020-6-3-159-168

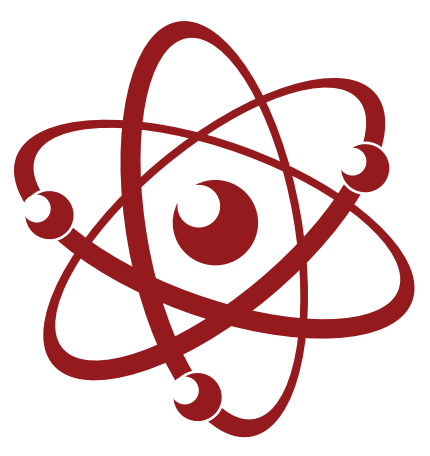

(C) И.С. Фишман, 2020 г.

\section{ПОСТАНОВКА ПРОБЛЕМЫ}

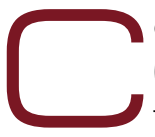

егодня, наряду с традиционными функциями университета (обучение студентов и осуществление научно-исследовательской деятельности), общество ставит перед ним задачу содействия социальному, экономическому и культурному развитию территории, на которой университет располагается. Этот запрос обозначен в литературе как третья миссия университета и реализуется посредством передачи академических и прикладных знаний (технологий и инноваций) для решения разнообразных задач социума и организации сотрудничества университета с государственными и частными предприятиями. На практике и то, и другое осуществляется путем коммерциализации научных и научно-технологических разработок.

В России работа по реализации данной миссии только начинается. Несмотря на то, что перед отечественной высшей школой не сегодня поставлена задача коммерциализации научных исследований, этот процесс сталкивается с существенными трудностями, одной из причин которых является неразвитость институциональных механизмов посредничества между университетами и бизнесом. 
Создаваемые в российских вузах (и при них) коммерческие структуры, как правило, не реализуют названную функцию. В связи с этим представляется актуальным анализ зарубежного опыта и введение в российский научный оборот результатов анализа зарубежными специалистами опыта деятельности структур, аффилированных университетам.

Данная работа посвящена вопросам возникновения и функционирования структур, занимающихся передачей академических знаний, технологизацией инноваций и аффилированных университетам США и Европы, а также механизмам коммерциализации университетских разработок, стимулирования и поддержки этого процесса. Именно этот зарубежный опыт создания институциональных посредников целесообразно учитывать в процессе коммерциализации инноваций отечественными университетами.

\section{ПРАКТИКА ЗАРУБЕЖНЫХ УНИВЕРСИТЕТОВ}

Для скорейшего включения в процесс передачи технологий и инноваций и реализации так называемой «третьей миссии» университеты в течение последних двух десятилетий энергично выстраивают связи с промышленным производством путем создания посреднических структур. Соответственно, более двух десятилетий структуры, занимающиеся коммерциализацией продуктов и технологий, созданных университетами, находятся в центре внимания исследователей [1]. Специалисты наблюдают за ростом, развитием и результативностью деятельности университетских структур и проводят сравнение с другими институциями, занимающимися коммерциализацией инновационных продуктов и разработок.

Наиболее распространенными среди них являются центры по передаче технологий (technology transfer offices, TTOs), университетские инкубаторы (university incubators, Uls) и совместные исследовательские центры (collective research centers, CRCs). Абсолютное большинство авторов не видят существенной разницы между этими организациями и обозначают их единым термином «побочный продукт университета»или «структура, отпочковавшаяся от университета» (university spin-offs,
USOs). Однако Villani и др. [2] различают три указанных структуры по назначению. Они считают, что центры по передаче технологий (TTOs) создаются для коммерциализации интеллектуальных разработок путем лицензирования, оказания консультативных услуг для исследователей, потенциальных изобретателей и представителей ближайшего сообщества. Инкубаторы (Uls) ставят цель вырастить новую университетскую структуру, оказывая помощь в администрировании, маркетинговой и коммерческой деятельности организации на начальном этапе ее функционирования, и связать университетский старт-ап с предпринимательской фирмой. Совместные же исследовательские центры (CRCs) создаются для того, чтобы запустить исследовательские проекты вместе с промышленными производствами и оказать содействие во внедрении готовых для коммерциализации продуктов или услуг. Rasmussen и др. [3] предлагают еще одну разновидность рассматриваемой структуры, так называемый внутренний фонд посевного финансирования (inner seed fund, ISF), определяя его функционал как поддержку запуска исследования и расширения рыночной ниши, которое может привести к появлению устойчивого внешнего финансирования.

Munari и др. [4] рассматривают посевные фонды (university seed funds, USFs), ориентированные на университеты и иные общественные исследовательские организации (public research oganisations, PROs), и их взаимодействие с USOs в Европе.

Данные фонды в Европе являются гетерогенными по многим основаниям, таким как управление, структура фонда, размеры и тип предоставляемого финансирования, а также направления инвестирования. Первая характеристика касается взаимоотношений фонда и университетов (и/или общественных исследовательских организаций), в частности того, какая организация отвечает за управление фондом. Авторы различают фонды, управляемые «изнутри»: университетами/PRO, и фонды, управляемые «извне»: группами частных инвесторов, национальными или региональными общественными агентствами, отвечающими за инновации и трансфер технологий. К группе 
внутренне управляемых фондов относят фонды, управляемые напрямую университетскими (PRO) офисами, ответственными за трансфер технологий или подобными им структурами. В этом случае университеты также формируют часть первоначального капитала фонда, и эта инвестиция, в большинстве случаев, дополняется другими, чаще из общественных источников, реже - из частных. Примерами USFs, которые можно включить в данную категорию, являются Imperial Innovation Fund of Imperial College в Великобритании, Baekeland Fonds of the University of Ghent в Бельгии, Inserm Transfert во Франции, Fraunhofer Ventures of Fraunhofer Institute в Германии. Как правило, такие внутренне управляемые фонды связаны с отдельным университетом или PRO, однако можно встретить и отдельные случаи, когда, в попытке достичь критической массы как с точки зрения управления фондом, так и базового объема сделок, фонды управляются двумя или большим количеством университетов. Примерами могут служить Wyern Seed Fund в Великобритании, созданный как совместное предприятие University of Southampton и University of Bristol, и KTH Chalmers Fund in Sweden, созданный для поддержки новых предприятий университета Chalmers и КТН университета.

Напротив, внешне управляемые фонды управляются частными инвесторами или общественными агентствами, часто в сотрудничестве с одним или более партнерскими университетами/PROs. Исследователи считают, что в эту категорию необходимо включать посевные фонды, в которые университеты (или PROs) финансово вовлечены в качестве партнеров с ограниченной ответственностью, но не участвуют напрямую в активном управлении предприятием. Это случай QBIC Fund в Бельгии (включающий Ghent University, Vrije Universiteit Brussel $и$ University of Antwerp), Venture Partners, $и$ Cascade Fund в Великобритании (включающий университеты Surrey, Brunel, Reading, Sussex и Royal Holloway). В эту группу следует также включать фонды, установившие формальное или неформальное сотрудничество с университетами и PROs, даже если они не используют прямого привлечения капитала исследовательских организаций. Здесь примером является португальский фонд Portugal
Ventures, TT Seed Fund, управляемый ТT Venture в Италии, и австрийский UniVenture Fund.

Необходимо отметить, что примерно 20-25\% фондов являются внутренне управляемыми (т.е. управляются университетами), тогда как 75-80\% управляются внешними субъектами. Таким образом, в Европе меньшинство посевных фондов, ориентированных на университеты/PROs являются «чистыми USFs», в то время как существенное большинство фондов имеют гибридные и более свободные структуры управления.

Еще одной важной структурной характеристикой USFs является величина фонда. По существующим данным, в среднем европейские USFs имеют небольшую начальную величину. Среднее значение величины фонда - порядка 30 млн. евро, а медианное - порядка 16,5 млн. евро. При этом фонды, управляемые «внутренне», т.е. университетами/PROs, имеют меньшую величину (в среднем, 10-11 млн. евро). По мнению авторов, для создания такого типа фондового инструмента требуются относительно меньшие стартовые инвестиции по сравнению с другими типами финансовых инструментов посевного вида и инструментов финансирования на ранних стадиях. USFs преимущественно используют акционерные инвестиции в стартапы. Описано лишь несколько частных случаев, когда используются гибридные формы инвестиций, такие как сочетание предпосевного гранта и акционерных (долевых) инвестиций или сочетание долевых инвестиций и кредита.

Другой важной характеристикой для классификации USFs является их инвестиционная стратегия. Авторы отмечают различные подходы с точки зрения направления инвестиций в компании определенного типа, технологический или индустриальный секторы экономики и географические области. В рамках первого направления анализа можно выделить USFs, которые намеренно инвестируют только в университетские и PRO spin-offs (или «трансляционные» программы финансирования, которые предусматривают инвестиции только в проекты, порождаемые университетами/PROs), и фонды, которые, в дополнение к университетским и PRO spin-offs, также инвестируют в другие типы новых компаний. Подавляющее большинство внутренне управляемых USFS относятся 
к первой категории, в то время как внешне управляемые имеют более широкую направленность инвестирования.

Еще одним важным аспектом анализа инвестиционной стратегии фондов является их фокусировка на технологическом секторе или географическом районе. Обе эти позиции связываются с показателями успешности венчурных фондов. Что касается географической специализации, то в ситуации, когда USFs создаются для коммерциализации технологий, разрабатываемых в определенных университетах, они, как правило, инвестируют преимущественно в территории, где университеты расположены. К тому же, они часто субсидируются и поддерживаются региональными агентствами инноваций или развития, поэтому неудивительно в этих случаях обнаружить географические ограничения в финансировании.

Существуют источники, которые свидетельствуют о том, что университетские центры трансфера технологий демонстрируют более высокую эффективность по сравнению с другими предпринимательскими фирмами, не связанными с университетами [5]. Ряд авторов, однако, предлагают не рассматривать эти центры как уникальное явление, требующее отдельного анализа, так как, по их мнению, большинство характеристик университетских центров аналогичны характеристикам любых иных фирм, занимающихся коммерциализацией технологий. Так, структуры обоих типов тратят существенные ресурсы на осуществление научно-исследовательской деятельности, работают в наукоемких отраслях науки, нанимают высокообразованный технический персонал. Фактически не привязанные к университету фирмы могут быть как независимыми, так и аффилированными той или иной компании или корпорации (холдинг, консорциум, конгломерат, трест) и заниматься коммерциализацией наработок родительской организации. Сравнение указанных структур Mathisen [6] предлагает осуществлять по трем критериям: венчурное финансирование организации, инновативность организации, развитие и эффективность организации.

Большинство частных венчурных фондов (venture capitalists, VCs) предпочитают инвестировать в структуру, аффилированную корпоративной организации, а не университету.
Исследователи Munari, Wright и др. [4, 7] отмечают три потенциальных риска. Во-первых, деятельность организаций, отпочковавшихся от университета, требует постоянного контроля из-за традиционной слабости управленческой команды. Во-вторых, их деятельность предполагает более дальний инвестиционный горизонт и, в-третьих, более низкий уровень доходов за сравнимый период. В то же время исследователи Munari, Toole, Yague-Perales, Zhang [8-11] указывают на то, что аффилированной университету структуре легче получить финансирование от венчурных фондов на этапе становления, если эти фонды частично или полностью государственные, так как последние предпочитают организации, имеющие прочные связи с университетами.

По критерию инновативности лидируют организации, имеющие прочные связи с университетом, так как, преимущественно, работают над продуктами, имеющими перспективы получить патент $[12,13]$. Эти организации уделяют основное внимание исследованиям, нанимают более квалифицированных сотрудников и могут полноценно использовать материальные ресурсы университета. Успешность инновационной деятельности структур, аффилированных университетам, объясняется их территориальной близостью к университетам и прочными партнерскими отношениями, им нет равных во внедрении инноваций, защищенных патентами [13]. Благодаря поддержке государства и общества эти организации демонстрируют более оптимистичное поведение и до последнего пытаются отработать идею, которая кажется однозначно провальной другим организациям, занятым коммерциализацией инноваций [14].

Одни исследователи [9, 11], считают, что у организации, аффилированной университету, шансов выжить больше, другие [15] - придерживаются противоположной точки зрения. Ряд исследователей: Ensley и Hmieleski, Salvador [16, 17] отмечают низкую доходность организаций, аффилированных университетам по сравнению с другими организациями, занимающимися технологизацией инноваций. Исследователи OrtinAngel и Vendrell-Herrero [18] объясняют низкую доходность связанных с университетом организаций тем, что им необходимо больше времени, чтобы превратить инновации в прибыльный 
бизнес. У исследователей нет единого мнения и по поводу роста объема продаж. Lowe и Ziedonis; Yague-Perales и March-Chorda [14, 10] считают, что он выше у структур, аффилированных университетам, тогда как Ensley и Hmieleski; Wright и др. [16, 7] утверждают, что рост продаж у них ниже.

Из-за своего «академического происхождения» структуры, связанные с университетами (USOs), как правило, разрабатывают продукты в меньшей степени готовые к внедрению, а члены команды обладают меньшими, чем у сотрудников фирм, аффилированных корпоративным организациям (corporate spin-offs, CSOs), умениями в области развития бизнеса. Однако USOs значительно больше выигрывают у CSOs, если все же приобретают эти умения. Опыт промышленного производства повышает эффективность деятельности USO, в свою очередь, предпринимательский опыт и доступ к ресурсам, которыми наделяют CSO создавшие их организации, дают им больше, чем USOs [15]. Y USOs более гомогенные управленческие команды, что ведет к меньшей степени сплочённости в коллективе, видение руководства не разделяется всеми членами коллектива и, как следствие, возникает значительное количество личностных конфликтов [16]. Это означает, что USO уступает CSO в аспекте сплоченности команды и групповой динамики.

CSOs развиваются быстрее, когда коммерциализирует узкоориентированную технологию, основывающуюся на неявном, «опытном» знании, отличном от технических знаний компании, породившей данную структуру. USOs же демонстрируют большую степень успешности в ситуациях коммерциализации не принципиально новых технологий широкого применения. Структуры, выросшие из корпоративных организаций, развиваются быстрее, если располагаются вблизи университетов, ориентированных на коммерциализацию своих идей [19].

USOs представляют существенную часть рынка высокотехнологичных компаний, осуществляющих первичное публичное предложение своих акций (Initial Public Offerings, IPOs). Это особенно очевидно в странах Европы с более развитой экономикой и, преимущественно, в биомедицинских отраслях [20].
Отмечается также, что USOs получают более высокую, чем CPOs, оценку стоимости в процессе IPO, особенно если ученые входят в управленческую команду организации [20]. Однако долгосрочная финансовая отдача от деятельности аффилированных университетам организаций ниже, чем от организаций, аффилированных с бизнесом [21].

USOs приобретаются другими субъектами рынка чаще, но гораздо реже сами приобретают фирмы $[12,21]$. USOs с гораздо большей готовностью приобретаются зарубежными компаниями, особенно если эти структуры связаны с престижными университетами, имеющими международный авторитет.

Аффилированные университету структуры (USOs) с большей долей вероятности получают финансирование от венчурных фондов или становятся открытыми для инвестиций, если заняты коммерциализацией защищенной патентом интеллектуальной собственности. Наличие патентов - однозначный позитивный сигнал для инвесторов и всех заинтересованных сторон, считают целый ряд авторов: Miozzo и DiVito; Stephan; Mueller и др. [13, 22, 23], так как USOs с патентами коммерциализируют явные изобретения. Фирмы же, ориентированные на оказание консультативных услуг, разработку программных продуктов или организацию исследований, должны затратить больше усилий, чтобы получить финансирование из венчурных фондов и суммы эти существенно меньше [4]. При этом характер знания (подтвержденные патентом изобретения или неявное знание), которое фирма доводит до продукта, не влияет на развитие организации и, в частности, на рост занятости.

Как новизна технологии, так и степень защиты интеллектуальной собственности повышают шансы организации на выживание, однако этот эффект более выражен на сегментированных рынках. Работа с непатентованными, кардинально новыми технологиями - это вызов для организации и для его преодоления критичными становятся наличие стабильного финансирования фирмы и доступ к дополнительным ресурсам через налаженные партнерские отношения со сторонними организациями. Защита интеллектуальной собственности зачастую является критическим предварительным условием роста, 
потому что USO не имеют контроля над конечным потребительским продуктом и потому, что патенты увеличивают возможность получения дополнительной прибыли в стратегических партнерствах [24].

USOs, которые коммерциализируют запатентованную технологию, содержащую высокую степень новизны, могут успешно работать на рынке технологий, продавая права на использование своих технологий другим фирмам, отмечают Gans и Stern [25]. Используя такую бизнес-модель, USOs тратят большую часть своих ресурсов на исследовательскую деятельность, зарабатывая, прежде всего, на перепродаже своих прав на интеллектуальную собственность. Развитые промышленные структуры приобретают такие биомедицинские технологии, поддерживая, тем самым, указанную бизнес-модель. Сильная защита от заимствования технологий также создает большие стимулы для работы на рынке технологий.

Привлечение венчурного капитала является фактором, прогнозирующим последующий рост фирмы, благодаря доступу к важнейшим финансовым ресурсам и вкладу венчурных капиталистов в человеческий и социальный капитал, констатируют Miozzo и DiVito [22]. Чем в большем количестве партнерств состоят USOs, тем более устойчив их коммерческий успех. Структуры, объединяющие исследовательскую деятельность и производство, приносят больший доход, чем фирмы, имеющие узкую специализацию. USOs, созданные как совместные предприятия с промышленностью, прекрасно справляются С трудностями в процессе становления и развития и демонстрируют высокую эффективность, утверждают Munari и Toschi [8], так как лучше распознают возможности рынка, поддерживают легитимность структуры и имеют доступ к критически важным ресурсам и возможностям.

\section{ОБСУЖДЕНИЕ И ВЫВОДЫ}

Подавляющее большинство авторов считают университетские структуры (как посреднические, так и фонды) важным и экономически устойчивым механизмом передачи новых технологий промышленности. Утверждается также, что все эти структуры ускоряют экономическое развитие территорий путем создания новых наукоемких рабочих мест, и увеличивают налоговые поступления в местные бюджеты. Ряд авторов отмечают опосредованные эффекты от распространения новых технологий, которые усиливают экономический и технологический потенциал региона. Исследователи подчеркивают, что создаваемые структуры важны с точки зрения реализации общественных потребностей, так как берутся за коммерциализацию таких научных результатов, которые могли бы остаться невостребованными. Ряд авторов отмечают последовательное увеличение поддержки академического предпринимательства как со стороны университетов, так и государственных организаций. В пример приводятся Genentech, Lycos и Google - фирмы, которые начали свою более чем успешную коммерческую деятельность с применения теоретических разработок и технологий, созданных в результате взаимодействия с университетами и государственными исследовательскими организациями. В некоторых отраслях промышленности, например, биотехнологической, структуры, отпочковавшиеся от университетов, составляют большую часть всех старт-ап бизнесов [20], в европейских же высокотехнологичных отраслях промышленности их четверть [21]. На региональном уровне влияние структур, аффилированных университетам, также достаточно велико. Так организации, занятые коммерциализацией исследований и технологий на базе Оксфордского университета, обеспечивают 3,5\% от уровня занятости местного населения [14], организации Канады, занимающиеся превращением наработок университетов в коммерческие продукты, имеют чистую приведенную стоимость, значительно превышающую суммарный размер инвестиций в исследования в смежных научных областях [26]. В 2016 г. в США были основаны более тысячи фирм, занимающихся трансфером инноваций и аффилированных университетам, а всего с 1995 г. их было создано, по меньшей мере, 11000 . Однако часть исследователей утверждают, что количество указанных организаций существенно занижено из-за сложностей в получении информации об их существовании и недоопределенности самого феномена USO. 
Тем не менее, ряд исследователей сомневаются в том, что университетские центры оправдывают внимание и поддержку общества. Утверждается, что, преимущественно, это очень маленькие фирмы, которые демонстрируют крайне незначительный рост и вносят небольшой вклад в экономику территории.

Обобщая источники, можно сделать вывод, что, благодаря государственному финансированию и общественной поддержке, USOs демонстрируют более высокую выживаемость по сравнению с CSOs. Они очень активно используют государственные программы помощи, в частности, являются предпочтительными реципиентами для государственных венчурных фондов. Аффилированные университетам структуры дольше остаются обитателями бизнес-инкубаторов и являются бенефициарами фондов, поддерживающих научно-исследовательские и опытно-конструкторские разработки. Однако следует еще раз подчеркнуть, что частные венчурные фонды охотнее финансируют организации, связанные с бизнес-структурами. Причинами этого могут стать избыточная представленность USOs в определенных отраслях промышленности и активное их финансирование государственными венчурными фондами.

Аффилированные университетам структуры являются и более инновативными, чем фирмы, аффилированные корпоративным организациям, так как имеют большее количество защищенных патентами изобретений, научно-исследовательских и опытно-конструкторских разработок, значительное количество высокообразованных сотрудников. Кроме того, они поддерживают, как было сказано выше, прочные связи со своим университетом и уровень инновативности напрямую зависит от степени географической близости структуры, отпочковавшейся от университета, и взаимной интеллектуальной вовлеченности в решение поставленных задач.

Выводы, связанные с развитием и эффективностью организации не столь однозначны: аффилированные университетам структуры демонстрируют более высокий уровень выживаемости и более высокую оценку ликвидности, однако имеют более низкую рентабельность.

Этот факт пока что не находит объяснения и заставляет специалистов анализировать процессы возникновения и развития организаций, занимающихся коммерциализацией знаний, а также причины их успешности или неудач.

Также следует отметить, что USOs - высоко инновационные фирмы и могут коммерциализировать и конкретные изобретения, и более неявное знание, полученное из научных исследований. Однако они получают большее финансирование, если их интеллектуальная собственность защищена патентом. По мнению большинства исследователей, самой большой сложностью для фирмы будет преобразование ее технологических активов в рыночные предложения. Это особенно сложно на устоявшихся рынках, и в случае, если у фирмы нет партнерских отношений с действующими субъектами рынка.

Налаженные связи с инвесторами и промышленными структурами являются значимыми факторами для роста, развития и эффективного функционирования фирмы. Успешные фирмы трансформируют свои прочные академические связи в широкую сеть бизнес-контактов. Более того, партнерства с бизнесом признаются значимыми для USOs, так как позволяют преодолеть недостаточность ресурсов и обеспечивают доступ к дополнительным активам. Однако долгосрочные последствия таких партнерств пока не изучены. Известно, что создание совместных предприятий с промышленными партнерами обеспечивает быстрый и продуктивный старт фирмы, однако ее будущее развитие будет связано только с этим бизнес-партнером, что не во всех случаях будет приносить желаемый эффект фирме.

Анализ зарубежных источников показывает, что в практике университетов активно развивается деятельность разнообразных посреднических структур для выполнения функций трансфера знаний и технологий в практику, в том числе Центры по передаче технологий (TTOs), Университетские инкубаторы (Uls), Совместные исследовательские центры (CRCs), Посевные фонды (USFs) и другие организации.

Фиксируется несколько видов посевных фондов, ориентированных на университеты: фонды, управляемые «изнутри» (20-25\% от всех европейских фондов), фонды, управляемые «извне», в том числе группами частных инвесторов, национальными или региональными общественными агентствами, отвечающими 
за инновации и трансфер технологий (75-80\% европейских фондов).

Большинство экспертов солидарны в том, что посреднические структуры и фонды ускоряют экономическое развитие территорий путем создания новых наукоемких рабочих мест и увеличивают налоговые поступления в местные бюджеты.

Сравнение эффективности деятельности структур по трансферу технологий, созданных в университетах, и вне их, показывает, что университетские центры трансфера технологий более эффективны. Благодаря государственному финансированию и общественной поддержке, они демонстрируют более высокую выживаемость и уровень инновационности, чем фирмы, аффилированные с корпоративными организациями. В то же время, в ряде исследований приведены данные о том, что университетские структуры, хотя и отличаются более высокой ликвидностью, однако менее рентабельны по сравнению с аналогичными структурами, аффилированными с бизнесом.

Аффилированные университетам структуры очень активно используют государственные программы помощи, дольше остаются обитателями бизнес-инкубаторов и являются бенефициарами фондов, поддерживающих научно-исследовательские и опытно-конструкторские разработки. При этом частные венчурные фонды охотнее финансируют организации, связанные с бизнес-структурами. Аффилированные университетам структуры являются более инновативными, чем фирмы, аффилированные корпоративным организациям, так как имеют большее количество защищенных патентами изобретений. Аффилированные университетам структуры демонстрируют более высокий уровень выживаемости и более высокую оценку ликвидности, однако имеют более низкую рентабельность.

Аффилированные университетам структуры высоко инновационные фирмы и могут коммерциализировать и конкретные изобретения, и более неявное знание, полученное из научных исследований. Но, по мнению большинства исследователей, самой большой сложностью для такой фирмы является преобразование ее технологических активов в рыночные предложения, что особенно сложно на устоявшихся рынках, и в случае, если у фирмы нет партнерских отношений с действующими субъектами рынка.

\section{ЛИТЕРАТУРА}

1. Mathisen M., Rasmussen T.E. (2019) The development, growth, and performance of university spinoffs: a critical review // The Journal of Technology Transfer. 1-48.

2. Villani E., Einar Rasmussen E., Grimaldi R. (2017) How intermediary organizations facilitate universityindustry technology transfer: A proximity approach. Technological forecasting and social change // An international journal. 86-102.

3. Rasmussen E., Moen O., Gulbrandsen M. (2006) Initiatives to promote commercialization of university knowledge // Technovation. 26:518-533.

4. Munari F., Pasquini M., Toschi L. (2015) From the lab to the stock market? The characteristics and impact of university-oriented seed funds in Europe // Journal of Technology Transfer. 40:948-975.

5. Shane S. (2004) Academic entrepreneurship university spinoffs and wealth creation / Cheltenham: Edward Elgar Publishing.

6. Mathisen M.T. (2017) The growth of research-based spin-offs: Unleashing the value of academic entrepreneurship / Norwegian University of Science and Technology. 224 p.
7. Wright M., Lockett A., Clarysse B., Binks M. (2006) University spin-out companies and venture capital // Munari Research Policy. 35:481-501.

8. Munari F., Toschi L. (2011) Do venture capitalists have a bias against investment in academic spinoffs? Evidence from the micro- and nanotechnology sector in the UK // Industrial and Corporate Change. 20:397-432.

9. Toole A.A., Czarnitzki D. (2007) Biomedical academic entrepreneurship through the SBIR program // Journal of Economic Behavior \& Organization. 63:716-738.

10. Yague-Perales R.M., March-Chorda I. (2012) Performance analysis of research spin-offs in the Spanish biotechnology industry // Journal of Business Research. 65:1782-1789.

11. Zhang J.F. (2009) The performance of university spin-offs: An exploratory analysis using venture capital data // Journal of Technology Transfer. 34:255-285.

12. Meoli M., Paleari S., Vismara S. (2013) Completing the technology transfer process: M\&As of science-based IPOs // Small Business Economics. 40:227-248. 
13. Stephan A. (2014) Are public research spin-offs more innovative? // Small Business Economics. 43:353-368.

14. Lowe R.A., Ziedonis A.A. (2006) Overoptimism and the performance of entrepreneurial firms // Management Science. 52:173-186.

15. Wennberg K., Wiklund J., Hellerstedt K., Nordqvist M. (2011) Implications of intra-family and external ownership transfer of family firms: short-term and long-termperformance differences // Strategic Entrepreneurship Journal. 5:352-372.

16. Ensley M.D., Hmieleski, K.A. (2005) A comparative study of new venture top management team composition, dynamics and performance between university-based and independent start-ups // Research Policy. 34:1091-1105.

17. Salvador E. (2011) Are science parks and incubators good «brand names» for spin-offs? The case study of Turin // Journal of Technology Transfer. 36:203-232.

18. Ortin-Angel P., Vendrell-Herrero F. (2014) University spin-offs vs. other NTBFs: Total factor productivity differences at outset and evolution // Technovation. 34:101-112.

19. Colombo M. G., D'Adda D., Piva E. (2010) The contribution of university research to the growth of academic start-ups: An empirical analysis // Journal of Technology Transfer. 35:113-140.
20. Bonardo D., Paleari S., Vismara S. (2011) Valuing university-based firms: The effects of academic affiliation on IPO performance // Entrepreneurship Theory and Practice. 35:755-776.

21. Bonardo D., Paleari S., Vismara S. (2010) The M\&A dynamics of European science-based entrepreneurial firms // Journal of Technology Transfer. 35:141-180.

22. Miozzo M., DiVito L. (2016) Growing fast or slow?: Understanding the variety of paths and the speed of early growth of entrepreneurial science-based firms // Research Policy. 45:964-986.

23. Mueller C., Westhead P., Wright M. (2012) Formal venture capital acquisition: can entrepreneurs compensate for the spatial proximity benefits of South East England and 'star' golden-triangle universities? // Environment and Planning. 44:281-296.

24. Lubik S., Garnsey E. (2016) Early business model evolution in science-based ventures: The case of advanced materials // Long Range Planning. 49:393-408.

25. Gans J.S., Stern S. (2003) The product market and the market for «ideas»: Commercialization strategies for technology entrepreneurs // Research Policy. 32:333-350.

26. Vincett P.S. (2010) The economic impacts of academic spin-off companies, and their implications for public policy // Research Policy. 39:736-747.

\title{
Информация об авторе
}

Фишман Ирина Самуиловна - кандидат педагогических наук, доцент, ведущий научный сотрудник, Самарский филиал Российской академии народного хозяйства и государственной службы при Президенте PФ; Scopus Author ID: 57195359893, ID: РИНЦ 5580-8073 (Российская Федерация, 443056, г. Самара, пр. Масленникова, д.37; e-mail: fishman-is@ranepa.ru).

\section{I.S. FISHMAN,}

Samara branch of the Russian Presidential Academy of National Economy and Public Administration (Samara, Russian Federation; e-mail: fishman-is@ranepa.ru)

\section{COMMERCIALIZATION OF INNDVATION BY WESTERN UNIVERSITIES}

\author{
UDC: 33 \\ https://doi.org/10.22394/2410-132X-2020-6-3-159-168
}

Abstract: It is shown that the vast majority of foreign researchers consider university structures to be an important and economically sustainable mechanism for the transfer of new industrial technologies, that these structures accelerate the economic development of territories by creating new knowledge-intensive (high-tech) jobs, and increase tax revenues to local budgets. Thanks to government funding and public support, university research structures demonstrate higher survival rates compared to firms affiliated to corporate organizations.

The structures affiliated to universities are very active in using state aid programs, remain residents of business incubators for longer, and are beneficiaries of funds supporting research and development. Private venture funds are more likely to finance them. University-affiliated structures are more innovative than firms associated with corporate organizations. These structures have a greater number of patented inventions, demonstrate a higher survival rate and a higher liquidity rating, but have lower profitability.

University-related structures are innovative firms and can commercialize both specific inventions and more implicit knowledge obtained as a result of scientific research. It is noted that the biggest challenge for such a firm is the transformation of technological assets into marketable offers. 
Keywords: University spin-offs, technology/innovation transfer, commercialization of innovation by universities, Technology Transfer Office, University Incubators, Collective Research Centers, Inner Seed Funds

For citation: Fishman I.S. Commercialization of Innovation by Western Universities. The Economics of Science. 2020;6(3):159-168. (In Russ.) https://doi.org/10.22394/2410-132X-2020-6-3-159-168

\section{REFERENCES}

1. Mathisen M., Rasmussen T.E. (2019) The development, growth, and performance of university spinoffs: a critical review // The Journal of Technology Transfer. 1-48.

2. Villani E., Einar Rasmussen E., Grimaldi R. (2017) How intermediary organizations facilitate universityindustry technology transfer: A proximity approach. Technological forecasting and social change // An international journal. 86-102.

3. Rasmussen E., Moen O., Gulbrandsen M. (2006) Initiatives to promote commercialization of university knowledge // Technovation. 26:518-533.

4. Munari F., Pasquini M., Toschi L. (2015) From the lab to the stock market? The characteristics and impact of university-oriented seed funds in Europe // Journal of Technology Transfer. 40:948-975.

5. Shane S. (2004) Academic entrepreneurship university spinoffs and wealth creation / Cheltenham: Edward Elgar Publishing.

6. Mathisen M.T. (2017) The growth of research-based spin-offs: Unleashing the value of academic entrepreneurship / Norwegian University of Science and Technology. 224 p.

7. Wright M., Lockett A., Clarysse B., Binks M. (2006) University spin-out companies and venture capital // Munari Research Policy. 35:481-501.

8. Munari F., Toschi L. (2011) Do venture capitalists have a bias against investment in academic spinoffs? Evidence from the micro- and nanotechnology sector in the UK // Industrial and Corporate Change. 20:397-432.

9. Toole A.A., Czarnitzki D. (2007) Biomedical academic entrepreneurship through the SBIR program // Journal of Economic Behavior \& Organization. 63:716-738.

10. Yague-Perales R.M., March-Chorda I. (2012) Performance analysis of research spin-offs in the Spanish biotechnology industry // Journal of Business Research. 65:1782-1789.

11. Zhang J.F. (2009) The performance of university spinoffs: An exploratory analysis using venture capital data // Journal of Technology Transfer. 34:255-285.

12. Meoli M., Paleari S., Vismara S. (2013) Completing the technology transfer process: M\&As of science-based IPOs // Small Business Economics. 40:227-248.

13. Stephan A. (2014) Are public research spin-offs more innovative? // Small Business Economics. 43:353-368.

14. Lowe R.A., Ziedonis A.A. (2006) Overoptimism and the performance of entrepreneurial firms // Management Science. 52:173-186.
15. Wennberg K., Wiklund J., Hellerstedt K., Nordqvist M. (2011) Implications of intra-family and external ownership transfer of family firms: short-term and long-termperformance differences // Strategic Entrepreneurship Journal. 5:352-372.

16. Ensley M.D., Hmieleski, K.A. (2005) A comparative study of new venture top management team composition, dynamics and performance between university-based and independent start-ups // Research Policy. 34:1091-1 105.

17. Salvador E. (2011) Are science parks and incubators good «brand names» for spin-offs? The case study of Turin // Journal of Technology Transfer. 36:203-232.

18. Ortin-Angel P., Vendrell-Herrero F. (2014) University spin-offs vs. other NTBFs: Total factor productivity differences at outset and evolution // Technovation. 34:101-112

19. Colombo M.G., D'Adda D., Piva E. (2010) The contribution of university research to the growth of academic start-ups: An empirical analysis // Journal of Technology Transfer. 35:1 13-140.

20. Bonardo D., Paleari S., Vismara S. (2011) Valuing university-based firms: The effects of academic affiliation on IPO performance // Entrepreneurship Theory and Practice. 35:755-776.

21. Bonardo D., Paleari S., Vismara S. (2010) The M\&A dynamics of European science-based entrepreneurial firms // Journal of Technology Transfer. 35:141-180.

22. Miozzo M., DiVito L. (2016) Growing fast or slow?: Understanding the variety of paths and the speed of early growth of entrepreneurial science-based firms // Research Policy. 45:964-986.

23. Mueller C., Westhead P., Wright M. (2012) Formal venture capital acquisition: can entrepreneurs compensate for the spatial proximity benefits of South East England and 'star' golden-triangle universities? // Environment and Planning. 44:281-296.

24. Lubik S., Garnsey E. (2016) Early business model evolution in science-based ventures: The case of advanced materials // Long Range Planning. 49:393-408.

25. Gans J.S., Stern S. (2003) The product market and the market for «ideas»: Commercialization strategies for technology entrepreneurs // Research Policy. 32:333-350.

26. Vincett P.S. (2010) The economic impacts of academic spin-off companies, and their implications for public policy // Research Policy. 39:736-747.

\section{Author}

Fishman Irina Samuilovna - PhD in Education, Associate Professor, Leading Research Fellow, Samara branch of the Russian Presidential Academy of National Economy and Public Administration; Scopus Author ID: 57195359893 (Russian Federation, 443056, Samara, Maslennikov Prospect, 37; e-mail: fishman-is@ranepa.ru). 